für die Wohnungen Tuberkulöser. ${ }^{1}$ ) Die Verfasser kommen bezüglich des Desinfektionswertes des Autans zu demselben verneinenden Resultat wie wir betreffs des Formalins (s. weiter unten). In derselben Arbeit sind auch Versuche über die Wirksamkeit des Rohlysoforms gegen den Tuberkelbacillus mitgeteilt. Da das Ergebnis mit dem unserigen völlig übereinstimmt, so kann ich mich bei der Mitteilung unserer Versuche kurz fassen. Die Anordnung der Experimente, besonders die Behandlung der Sputa war die gleiche, wie bei den Versuchen über Formalindesinfektion ausführlich beschrieben. Die Untersuchung

Aus den Heilstätten Friedrichsheim und Luisenheim. (Direktor: Dr. Curschmann.)

\section{Ueber die Wirkung des Formaldehyds auf tuberkulöses Sputum.}

Von Medizinalpraktikant Albert Kaiser.

Auf die Mitteilung von Herrn Chefarzt Dr. Roepke (Melsungen), daß die Tuberkelbazillen, mögell sie im Sputumausstrich oder im Sputumballen auf Wäsche übertragen sein, durch 24 stündiges Einwirken einer 1\%igen oder durch 2 wölfstündiges Einwirken einer $2 \%$ igen Rohlysoformlösung sicher abgetötet werden, ${ }^{1}$ ) beabsichtigte die Direktion der Heilstätten Friedrichsheim und Luisenheim, dieses Desinfektionsverfahren ebenfalls für die Wäsche einzuführen. Ich wurde beauftragt, zunächst einmal die Wirkung des Lysoforms experimentell nachzuprüfen.

Es stand mir zu Beginn meiner Versuche nur Reinlysoform zur Verfügung. Von Anfang an ging mein Bestreben dahin, die Verhältnisse für die Versuche so zu gestalten, daß sie denen in der Praxis möglichst nahekamen.

Der Versuch 1 sollte zeigen, ob eine 1\% \% ige Lösung von Reinlysoform bei 24 stündiger Einwirkungsdauer auf geballtes Sputum eines Tuberkulösen Wirkung besitzt.

$\mathrm{Zu}$ diesem Zweek wurde das Sputum Th., das nach Gaffkys Skala Tuberkelbazillen VIII enthielt, in physiologischer Kochsalzlösung auf $2 \mathrm{ccm}$ gebracht, mit $2 \mathrm{ccm}$ einer $2 \%$ igen Reinlysoformlösung vermischt, nach 24 stündiger Einwirkungsdauer in Kochsalzlösung abgespült, zerrieben, etwas verdünnt und $1 \mathrm{ccm}$ der Verdünnung dem Meerschweinchen A., Gewicht $450 \mathrm{~g}$, am 18. Februar 1908 in die Bauchdecken subkutan injiziert. Das Sputum selbst sah nach der Einwirkung etwas trübe aus, war aber in Form und Konsistenz nicht merklich verändert.

Das Tier wurde am 2. April 1908 getötet, nachdem es bereits in der letzten Zeit stark abgemagert war. Der Sektionsbefund ergab: An der Einstichstelle ein Abszeß, in dessen Eiter einzelne Tuberkelbazillen gefunden wurden. In Lunge und Leber einzelne große Tuberkelknoten; die Milz war sehr stark tuberkulös.

Der zweite Versuch sollte prüfen, ob der Tuberkelbacillus, wenn er mit dem Sputum in dünner Schicht an der Wäsche angetrocknet ist, durch 24 stündige Einwirkung einer $1 \%$ igen Reinlysoformlösung vernichtet wird.

Es wurde das Sputum W., das nach Gaffkys Skala Tuberkelbazillen VIII enthielt, dünn auf mehrere Mulläppchen verstrichen und im Dunklen angetrocknet. Die Läppchen wurden 24 Stunden in 1 \% ige Reinlysoformlösung gebracht, darauf vorsichtig in physiologischer Kochsalzlösung abgewaschen, um das anhaftende I,ysoform $\mathrm{zu}$ beseitigen. Dann wurden sie in NaCl-Lösung tüchtig ausgeschüttelt, die Lösung schwach zentrifugiert, der Bodensatz etwas verdünnt und $1 \mathrm{ccm}$ dem Meerschweinchen B., Gewicht $430 \mathrm{~g}$, am 18. Februar 1908 in die Bauchdecken subkutan injiziert. Das Tier starb abgemagert am 31. März 1903. Sektionsbefund: Tuberkulöse Achseldrüsen. In der Lunge einige kleine tuberkulöse Herde. Milz suspekt. Leber und Nieren frei. Vergrößerte Mesenterialdrüsen.

Es waren also in beiden Versuchen die Tuberkelbazillen durch 24 stündige Einwirkung von 1\%iger Reinlysoformlösung nicht abgetötet worden. Da bei der Reinigung des Rohlysoforms zu Reinlysoform Formaldehyd eventuell verloren gehen könnte, infolgedessen Rohlysoform stärker desinfizierend wirken könnte als Reinlysoform, stellten wir später dieselben Versuche mit Rohlysoform an.

Noch während der Vornahme dieser Versuche veröffentlichten Dr. Kaufmann und cand med. Mietzsch aus der neuen Heilanstalt für Lungenkranke Schömberg die Ergebnisse ihrer Untersuchungen über den Desinfektionswert des Autans

1) Zeltschrift für Tuberkulose, Bd. 8 , Heft 3 nach der Desinfektion ergab stets nur Detritus, dagegen waren die Tuberkelbazillen immer gut färbbar nachzuweisen. Mischbakterien wurden mit Ausnahme eines Falles, in dem sich eine ganz blaß tingierte Coccenkolonie nach der Desinfektion zeigte, nie gefunden, obwohl die verwendeten Sputa vor der Desinfektion sie reichlich enthielten.

I. 24 stündige Desinfektion mit $1 \%$ iger Rohlysoformlösung.

A. Verwendung von geballtem Auswurf. 1. Auswurf Mü., nach Gaffky s Skala Tuberkelbazillen VIII, dem Meerschweinchen O., $400 \mathrm{~g}$ schwer, am 20. August $19081 \mathrm{ccm}$ in die Bauchdecken injiziert. Das Tier wurde am 8. Oktober 1908 getötet. Sektionsbefund: Gewicht $350 \mathrm{~g}$. In beiden Leistenbeugen große Drüsenpakete, Leber und Milz von großen tuberkulösen Knoten durchsetzt. Lungen einige Herde. Nieren frei.

2. Derselbe Auswurf dem Tier O. I am 20. August $19081 \mathrm{ccm}$ in die Bauchdecken injiziert. Exitus am 24. September 1908. Sektionsbefund: In der linken Leistenbeuge große, teilweise verkäste Drüsen. Im Ausstrich massenhaft bla $\beta$ tingierte Tuberkelbazillen und Detritus. Milz einige Herde. In der Leber verstreute große und kleine Knoten. In der Lunge, besonders in den Unterlappen, kleine Herde. Nieren ohne Befund.

B. Verwendung von an Leinwand in dünner Schicht an getrocknetem Auswurf. Auswurf H. nach Gaffky Tuberkelbazillen IX, dem Tier P., Gewicht $420 \mathrm{~g}$, am 27. August $19081 \mathrm{ccm}$ injiziert. Das 'Tier wurde am 8. Oktober 1908 getötet. - Sektionsbefund: Starke Abmagerung. Gewicht $330 \mathrm{~g}$. Große Leistendrüsen beiderseits, Lunge, Leber, Milz mäßig stark tuberkulös. Nieren frei.

C. Verwendung von auf Glas in düner Schicht ausgestrichenem und angetrocknetem Auswurf. Dasselbe Sputum wie beim vorigen Versuch am 28. August 1908. Dem Tier Q., $340 \mathrm{~g}$ schwer, injiziert. Getötet am 8. Oktober 1908. Sektionsbefund: Gewicht $307 \mathrm{~g}$. Große Leistendrüsen beiderseits, links teilweise verkäst. Im Ausstrich reichlich gut gefärbte Tuberkelbazillen. Milz und Leber sind stark tuberkulös. In der Lunge finden sich einige kleine Herde. Nieren frei.

II. 12stündige Desinfektion mit 2\%iger Rohlysoformlösung.

A. Verwendung von geballtem Sputum. Auswurf We., nach Gaffk ys Skala Tuberkelbazillen VIII, am 25. August 1908 dem Tier R., Gewicht $450 \mathrm{~g}$, in die Bauchdecken injiziert. Das Tier wurde am 8. Oktober 1908 getötet. - Sektionsbefund: Großes Drüsenpaket, teilweise verkäst, rechts und links kleinere Drüsen. Leber und Milı stark von tuberkulösen Knoten durchsetzt. In der Lunge einige kleine Herde. Nieren ohne Befund.

B. Verwendung von an Leinwand in dünner Schicht an getrocknetem Auswurf. Dasselbe Sputum We., am 27. August 1903 dem Tier S., Gewicht $430 \mathrm{~g}$, in die Bauchdecken injiziert. Das Tier wurde am 8. Oktober 1908 getötet. - Sektionsbefund: Infiltration der Einstichstelle. Große Leistendrüsen beiderseits. Milz großer tuberkulöser Tumor. In der Leber verstreute große und kleine, in der Lunge einige kleine Herde. Nieren frei.

C. Verwendung von auf Glas in dünner Schicht ausgestrichenem und angetrocknetem Auswurf. Dasselbe Sputum We., am 27. August 1908 dem Tier T., $420 \mathrm{~g}$ schwer, in die Bauchdecken injiziert. Das Tier wurde am 8. Oktober 1908 getötet. Sektionsbefund: Große Leistendrüsen beiderseits. Leber und Mil. einzelne tuberkulöse Herde. Lunge suspekt. Nieren ohne Befund.

Es wal also weder durch 24 stündige Einwirkung von $1 \%$ iger, noch durch 12 stündige Einwirkung einer $2 \%$ igen Reinlysoformlösung der Tuberkelbacillus im ballenförmigen Auswurf vernichtet worden. Aber auch das Rohlysoform hatte sich, mochten wir es in $1 \%$ iger Lösung 24 Stunden oder in $2 \%$ iger Lösung 12 Stunden auf Tuberkelbazillen enthaltendes Sputum einwirken lassen, ganz gleichgültig, ob wir ballenförmiges, in dünner Schicht an Leinwand angetrocknetes oder auf Glas ausgestrichenes Sputum verwandten, als unfähig er- 
wiesen, den Tuberkelbacillus im tuberkulösen Auswurf abzutöten. Denn alle Versuchstiere zeigten ausgebreitete Tuberkulose. Da das Rein- wie das Rohlysoform für die Versuche frisch in Originalpackung bezogen war, konnte ein Zweifel an der Güte der verwandten Präparate nicht bestehen, sondern das Fehlschlagen der Versuche dem Tuberkelbacillus gegenüber muß auf die Unwirksamkeit des wirksamen Prinzips im Lysoform, das ist das Formaldehyd, zurückgeführt werden.

Durch den Ausfall dieser Versuche hatte sich für uns die gestellte Frage verschoben, und es kamen uns Zweifel, ob überhaupt durch Formaldehyd, besonders bei der ja vielerorts eingeführten Formalindesinfektion der Tuberkelbacillus im tuberkulösen Sputum vernichtet wird. Wichtig für uns war diese Frage deshalb, weil ja in Heilstätten und Sanatorien fast allgemein die Krankenzimmer nit Formalin desinfiziert werden und das gleiche Verfahren zur Desinfektion von Decken, Teppichen und Betten angewandt wird. Aber auch für die Allgemeinheit ist es eine Frage von größter Tragweite, da ja auch durch die Gemeinden vielfach eine Desinfektion der von Phthisikern bewohnt gewesenen Räume angeordnet und vorgenommen wird, die fast überall mit Formalin geschieht. Die Frage also, ob von einem unreinlichen Tuberkulösen gebrauchte Gegenstände mit Formalin wirksam desinfiziert werden können, durfte von uns keinenfalls außer acht gelassen werden.

Bei der Desinfektion der von Tuberkulösen bewohnten Krankenzimmer und der von ihnen benutzten Gebrauchsgegenstände handelt es sich in erster Linie nicht darum, ob freie oder einzelne Tuberkelbazillen abgetötet werden, sondern ob in Sputum eingebettete vernichtet werden. Eine Desinfektion, die nur die Begleitmikroben tötet, ohne den Tuberkelbazillen zu schaden, kann sogar schlimmer sein als gar keine, da dann derTuberkelbacillus seine Wirkung ungestört und ohne Konkurrenz entfalten kann. Wenn die Formalindesinfektion ihren Zweck bei der Bekämpfung der Tuberkulose erfüllen und nicht direkt schaden soll, muß sie mit absoluter Sicherheit folgendes leisten. Sie muß Tuberkelbazillen in ballenförmigem Sputum, wie es sich in feuchten, dunklen Räumen lange Zeit halten kann, abtöten, muß ferner Sputum, das z. B. in Bettvorlagen, Sophaüberzügen etc. eingetrocknet ist, und endlich irgendwo an den Wänden oder dem Fußboden angètrocknetes Sputum sicher desinfizieren. $O b$ das Formalin das zu leisten vermag, war also zu untersuchen.

Die Versuche wurden in unserer eigens für Formalindesinfektion angelegten Desinfektionskammer vorgenommen, die einen Rauminhalt von $13,475 \mathrm{cbm}$ hat. Die zu desinfizierenden Sputa wurden mittags um $2 \mathrm{Uhr}$ in die Kammer gebracht und in verschiedener Höhe aufgestellt. Es wurden dann $200 \mathrm{ccm}$ $40 \%$ iges Formalin und $720 \mathrm{~g}$ Wasser mit dem Schneiderschen Apparat verdampft und die Sputa der Einwirkung des Formalindampfes bis früh um 8 , also 18 Stunden, überlassen. Auf die Zuleitung von Ammoniak verzichteten wir. Auf $1 \mathrm{cbm}$ Raum kamen also mehr als $5 \mathrm{~g}$ reines Formalin zur Verdampfung.

Zunächst brachten wir Sputum in Ballenform in etwas physiologischer Kochsalzlösung suspendiert in die Kammer, in der es in ganz flachen Schalen aufgestellt wurde. Nach der Desinfektion sah der Auswurf gallertig, etwas zerklüftet und schmierig aus. Im mikroskopischen Präparat fand sich Detritus, die vorher reichlich vorhandenen Coccen und Bakterien waren entweder ganz verschwunden oder doch nur sehr spärlich und schwach gefärbt nachzuweisen. Dagegen blieben die Tuberkelbazillen färbbar, wenn auch vielleicht etwas blasser als vor der Desinfektion. Oft zeigten sie eine vorher nicht beobachtete Körnelung. Die Sputa wurden nun in Kochsalzlösung abgespült, verrieben, etwa auf das Doppelte verdünnt und $1 \mathrm{ccm}$ der Verdünnung unter die Bauchdecken stets subkutan injiziert. Ich lasse nun die angestellten Versuche folgen.

1. Auswurf S. nach Gaffky, Skala Tuberkelbazillen VIII, dem Meerschweinchen C. Gewicht $430 \mathrm{~g}, 1 \mathrm{ccm}$ am 24. Juni 1908 in die Bauchdecken injiziert. Exitus am 10. Juli 1908. Die Sektion ergab: Croupöse Pneumonie der ganzen rechten Lunge, fettige Degeneration der Leber. Keine Zeichen von Tuberkulose.

1 b. Dasselbe Sputum auf Tier Cl., Gewicht $250 \mathrm{~g}, 1 \mathrm{ccm}$ am
24. Juli 1908 in die Bauchdecken injiziert. Exitus am 3. Septem ber 1908. Gewicht $435 \mathrm{~g}$. Sektionsbefund: Lunge einige kleine tuberkulöse Herde. Leber stark tuberkulös. Milz einige kleine Herde. Nieren frei.

2. Auswurf B. nach G affky, Tuberkelbazillen IX, dem Tier D., Gewicht $490 \mathrm{~g}$, am 30. Juni 1908 in die Bauchdecken injiziert. Exitus am 6. September 1908. Sektionsbefund: Tuberkulöse Leistendrüsen beiderseits. Milz stark tuberkulös. In der Leber einige große tuberkulöse Knoten, teilweise verkäst, sehr viele kleine Knötchen. In der Lunge einige tuberkulöse Herde. Nieren frei.

2 b. Dasselbe Sputum dem Tier D. 1, Gewicht $430 \mathrm{~g}, 1 \mathrm{ccm}$ am 30. Juni 1908 in die Bauchdecken injiziert. Exitus am 23. September 1908. Sektionsbefund: Tier stark abgemagert. Gewicht $300 \mathrm{~g}$. In der rechten Leistenbeuge großes tuberkulöses Drüsennaket. Zwischen der Einstichstelle in den Bauchdecken und der Torderfläche der Leber derbe fibröse Stränge. Leber völlig von ausgedehnten, teilweise verkästen tuberkulösen Inf $l$ ltrationen durchsetzt. Milz stark tuberkulös. In beiden Lungen einzelne große, teilweise verkäste Herde. Exsudative seröse Pleuritic. Nieren frei.

3. Auswurf M. nach Gaffky, Tuberkelbazillen IX, dem Tier E., $495 \mathrm{~g}$ schwer, am 2. Juli $19081 \mathrm{ccm}$ in die Bauchdecken injiziert. Exitus 10. August 1908. Sektionsbefund: Großer Abszeß in den Bauch decken an der Einstichstelle, in dessen Eiter Detritus und massenhafte Tuberkelbazillen nachweisbar sind. Tuberkulöse Leistendrüsen beiderseits. Leber völlig von tuberkulösen Infiltrationen und teilweise verkästen Knoten durchsetzt. Die Milz bildet einen sehr großen tuberkulösen Tumor. Lunge und Nieren sind frei.

$3 \mathrm{~b}$. Das gleiche Sputum auf Tier E. 1, Gewicht $495 \mathrm{~g}, 1 \mathrm{ccm}$ am 2. Juli 1908 in die Bauchdecken injiziert. Exitus am 9. August 1908. Sektionsbefund: Tier stark abgemagert. In der rechten Leistenbeuge große verkäste Drüsen. Im Ausstrich Detritus und massenhaft Tuberkelbazillen. Leber vollständig von tuberkulösen Infiltrationen und großen, teils verkästen Knoten durchsetzt. Im Messerrückenabstrich Detritus, verfettete Leberzellen und reichlich Tuberkelbazillen. Milz sehr großer tuberkulöser Tumor. Lunge und Nieren frei.

4. Auswurf Ba., Tuberkelbazillen aach G aff k y VIII, dem Tier F., Gewicht $410 \mathrm{~g}, 1 \mathrm{ccm}$ am 14. Juli 1908 in die Bauchdecken injiziert. Exitus am 2. September 1908. Sektionsbefund: Tier stark abgemagert. Gewicht $310 \mathrm{~g}$. Beiderseits tuberkulöse Leistendrüsen, links zum Teil verkäst. Lunge, besonders der linke Unterlappen von zahlreichen kleinen Herden durchsetzt. Leber und Milz stark tuberkulös. Nieren frei.

4 b. Das gleiche Sputum dem Tier F. 1., Gewicht $480 \mathrm{~g}, 1 \mathrm{ccm}$ am 14. Juli 1908 in die Bauchdecken injiziert. Exitus am 27. August 1908. Sektionsbefund: Tier enorm abgemagert. Gewicht 250 g. In beiden Leistenbeugen große Drüsenpakete. In der Lunge einzelne kleine Herde. Milz und Leber sehr stark tuberkulös. Plastische Peritonitis, besonders im kleinen Becken.

Zu dem Kontrollversuche für diese Versuchsreihe wurde das für die Versuche 4 und $4 \mathrm{~b}$ verwandte Sputum Ba. benutzt, weil es uns infolge seines enormen Reichtums an Mikroben verschiedenster Art geeignet schien, den Einfluß des Formalins auf diese zu prüfen. Das Sputum wurde in Kochsalzlösung verrieben, etwas verdünnt und $1 \mathrm{ccm}$ der Verdünnung in die Bauchdecken injiziert. Das Ergebnis war folgendes: Das Tier G., Gewicht $400 \mathrm{~g}$, am 17. Juli 1908 gespritzt. Exitus am 28. Juli 1908. Sektionsbefund: Großer Absze $B$ in den Bauchdecken. Eiter stinkend. Mikroskopisch Leuko. cyten, Detritus, massenhaft Mikroben, besonders Streptococcen, ganz vereinzelte, blaß gefärbte Tuberkelbazillen. Die ganze Bauch höhle mit Blut angefüllt, das jedenfalls aus einer beim Durchbruch des Abszesses in die Peritonealhöhle arrodierten Arterie stammt. Alle Organe sehr anämisch. Keine Organtuberkulose nachweisbar.

Tier G. I, $460 \mathrm{~g}$ schwer, injiziert am 17. Juli 1908. Exitus am 23. Juli 1908. Sektionsbefund: Großer Abszeß der Bauchdecken. Perforation in die Bauchhöhle. Eitrige Peritonitis. Im Eiter sehr reichlich Streptococcen, keine Tuberkelbazillen nachweisbar. Keine Tuberkulose der inneren Organe zu konstatieren.

Um nun nachzuprüfen, ob das Formalin imstande sei, an Wäsche etc. angetrocknetes Sputum wirksam zu desinfizieren, wurde das Sputum Mü. in ganz dünner Schicht auf Leinwand ausgestrichen, im Dunklen angetrocknet, 18 Stunden in der Desinfektionskammer mit $200 \mathrm{ccm}$ Formalin $+720 \mathrm{~g}$ Wasser desinfiziert, mit Kochsalzlösung ausgewaschen und $1 \mathrm{ccm}$ der erhaltenen Lösung dem Tier H., $450 \mathrm{~g}$ schwer, am 25. August 1908 in die Bauchdecken injiziert. Das Sputum enthielt nach Gaffkys Skala Tuberkelbazillen VIII. Das Tier wurde am 6. Oktober 1908 getötet. Sektionsbefund: $385 \mathrm{~g}$, in beiden Leistenbeugen tuberkulöse Drüsen. Mılz, Lunge stark, Leber schwach tuberkulös. Nieren frei.

Endlich galt es noch zu untersuchen, ob an Wänden etc. an- 
getrockneter Auswurf mit Formalin unschädlich gemacht werden könnte. Es wurde das Sputum Br. nach Gaffkys Skala Tuberkelbazillen VIII so dünn, daß es nach dem Trocknen eine gerade noch wahrnehmbare Schicht auf dem Glas bildete, auf eine Glasplatte ausgestrichen im Dunklen angetrocknet, 18 Stunden mit $200 \mathrm{ccm}$ Formalin $+720 \mathrm{~g}$ Wasser desinfiziert. Dann wurde das sputum abgekratzt, in Korhsalzlösung aufgeschwemmt und $1 \mathrm{ccm}$ dem Tier J., $480 \mathrm{~g}$ schwer, am 26. August 1908 in die Bauchdecken injiziert. Das Tier wurde am 6. Oktober 1908 getötet. Sektionsbefund: Tier ist stark abgemagert. Gewicht $345 \mathrm{~g}$. Beiderseits große, links teilweise verkäste Leistendrüsen, Lunge einige kleine tuberkulöse Herde. Leber und Milz stark tuberkulös. Nieren frei.

Nachdem sich also $200 \mathrm{ccm}$ Formalin trotz der langen Einwirkung von 18 Stunden als unwirksam gegen den Tuberkelbacillus erwiesen hatten, stellten wir die folgenden Versuche mit $400 \mathrm{ccm}$ Formalin $+1200 \mathrm{~g}$. Wasser, also pro Kubikmeter Raum mehr als $10 \mathrm{~g}$ reines Formalin, an. Im übrigen blieb die Anordnung der Versuche, was Zeitdauer der Desinfektion, Behandlung der verwendeten Sputa anbetrifft, völlig die gleiche, wie oben beschrieben. Makroskopisch wie mikroskopisch fand sich nach der 18 stündigen Desinfektion mit $400 \mathrm{ccm}$ Formalin derselbe Befund wie bei gleich lange dauernder Einwirkung von $200 \mathrm{ccm}$. Besonders waren die Tuberkelbazillen stets gut gefärbt nachweisbar.

Zunächst wurde wieder ballenförmiges Sputum verwendet. 1. Auswurf Bd, nach Gaffkys Skala Tuberkelbazillen VIII, wurde am 14. A ugust 1908 dem Meerschweinchen K., Gewicht $500 \mathrm{~g}$, in die Bauchdecken injiziert. Exitus am 18. September 1908 Sektionsbefund: Gewicht $450 \mathrm{~g}$. An der Einstichstelle ein großer AbszeB, in dessen Eiter massenhaft Tuberkelbazillen nachweisbar sind. Tuberkulö-e Drüsen in beiden Achseln und Leistenbeugen. Leber und Milz vollständig von tuberkulösen Infiltrationen durchsetzt, in der Lunge einige große Herde. Beiderseits großer seröser Pleuraerguß. Linke Niere verdichtig. rechte gesund.

1 b. Dasselbe Sputum am gleichen Tage dem Tier K. I, Gewicht $450 \mathrm{~g}$, injiziert. Das Tier wurde am 5. Oktober 1908 getötet Sektionsbefund: Gewicht $430 \mathrm{~g}$. In beiden Leistenbengen große, teilweise verkäste Drüsen. Die Milz bildet einen großen tuberkulösen Tumor. Leber und Lunge stark tuberkulös. Nieren frei.

2. Auswurf R., nach Gaff ky Skala Tuberkelbazillen VIII, am 18. August 1908 dem Tier L., $600 \mathrm{~g}$ Gewicht, in die Bauchdecken injiziert Exitus am 29. September 1908. Sektionsbefund: Enorme A bmagerung. Gewicht $300 \mathrm{~g}$. In beiden Leistenbeugen und Achselhöhlen große zum Teil verkäste Drüsen. Im Käse ziemlich reichlich gut färbbare Tuberkelbazillen. Lunge verdächtig, Leber stark, Milz mäßig tuberkulös. Nieren frei.

2b. Dasselbe Sputum am gleichen Tage dem Tier L. 1, $500 \mathrm{~g}$ Gewicht, injiziert. Das Tier wurde am 6. Oktober 1908 getötet. Sektionsbefund: Sehr starke Abmagerung. Gewicht $307 \mathrm{~g}$. Beiderseits große, teilweise verkäste Leistendrüsen. In der Leber und am Fundus der Gallenblase einige tuberkulöse Herde. Die Lunge ist stark tuberkulös. Milz und Nieren sind frei.

Das Sputum W., nach Gaffkys Skala Tuberkelbaxillen VIII, wurde in oben beschriebener Weise, auf Leinwand ausge. strichen, $18 \mathrm{Stunden}$ mit $400 \mathrm{ccm}$ Formalin $+1200 \mathrm{~g}$ Wasser desinfiziert und am 27. August 1908 dem Meerschweinchen M. in die Bauchdecken injiziert. Das Tier wog $420 \mathrm{~g}$. Exitus am 28. September 1908. Sektionsbefund: Starke Abmagerung. Gewicht $240 \mathrm{~g}$ In beiden Leistenbeugen große, teilweise verkäste Drtisen. Im Käse spärliche blaß tingierte Tuberkelbazillen. Milz suspekt. In der Leber einzelne große Knoten. Lunge stark tuberkulös. Nieren frei.

Dasselbe Sputum wurde endlich in ganz dunner Schicht a uf Glas angetrocknet und nach der Desinfektion am 27. A ugust 1908 dem Tier N., Gewicht $500 \mathrm{~g}$, injiziert. Das Tier wurde am 6. Oktober 1908 getötet. Sektionsbefund: Stark abgemagert. Große Leistendrüsen beiderseits. Milz stark, Leber wenig tuberkulö. Lunge verdächtig. Nieren frei.

Das Ergebnis der Versuche war also: Trotz der langen Einwirkung der Formalindesinfektion und der bei den letzten Versuchen auf mehr als $10 \mathrm{ccm}$ verdampftes Formalin pro Kubikmeter Raum gesteigerten Menge des Formalins waren in jedem Falle die Tuberkelbazillen nicht nur nicht getötet, sondern anscheinend nicht einmal in ihrer Virulenz merklich geschädigt worden. Von den 16 mit formalindesinfiziertem Auswurf injizierten Versuchstieren zeigten 15 das typische Bild schwerer, ausgebreiteter Tuberkulose. Das 16. Tier ging nach kurzer Zeit an einer Pneumonie zugrunde, scheidet also aus. (Vgl. Versuch 1, Tier C.) Da sich nicht nur bei Verwendung von. ballenförmigem Sputum, sondern auch bei Injektion von in ganz dünner Schicht auf Leinwand ausgestrichenem oder an Glas angetrocknetem Auswurf stets ausgebreitete Tuber kulose fand, so ist damit erwiesen, dab für den an sich gegen Formaldehyd resistenten Tuberkelbacillus eine feinste Schleimhülle genügt, um ihn vollständig jeder Einwirkung des Formalins zu entziehen. Es fällt also jede Einwirkung der Formalindesinfektion weg. Die Formalindesinfektion der von Phthisikern bewohnt gewesenen Räume muß demnach geradezu als eine Scheindesinfektion imponieren, die unter Umständen schlimmer sein kann als gar keine; denn anderseits ergaben die Versuche, daß Begleitbakterien im Sputum nicht gegen die Formaldehydwirkung geschützt sind, sondern vernichtet werden, wie der Versuch 4 und $4 \mathrm{~b}$ und die mit dem gleichen nicht desinfiziertem Sputum angestellten Kontrollen ergeben. Während die Tiere bei den Versuchen 4 und $4 \mathrm{~b}$ eine schwere, durch keine Mischinfektion gestörte Tuberkulose zeigten, gingen beide Kontrolltiere an Mischinfektion ein. Es kann nun immerhin die Frage entstehen, ob es als ein günstiger oder ungünstiger Vorgang aufzufassen ist, wenn nach der Formaldehydeinwirkung die Tuberkelbazillen sozusagen in Reinkultur zurückbleiben

Eine Stallinfektion der Versuchstiere ist auszuschlieben, da die verwandten Tiere einem vollständig gesunden, in der Anstalt selbst gezüchteten Stamme entnommen wurden und Tiere desselben Stammes bei anderweitigen Versuchen unter genau denselben äußeren Bedingungen tuberkulosefrei blieben.

Resümee. Für die praktische Anwendung ergibt sich aus Vorstehendem: Das Formaldehyd, mag man nun zur Desinfektion Reinlysoform, Rohlysoform, Formalin oder, wie Ka ufman und Mietzsch bewiesen haben, Autan verwenden, ist nicht imstande, den Tuberkelbacillus im Sputum abzutöten. Zur Desinfektion von Phthisikerwohnungen muB zum mindesten eine gründliche Reinigung mit Bürste und Scheuerlappen mi der Formaldehyddesinfektion einhergehen. Zur Desinfektion gröberer Sputumverunreinigungen werden nach wie vor Sublimat, Kresolseife oder Lysol trotz der mannigfachen Unbequemlichkeiten, die die Anwendung dieser Mittel mit sich bringt, herangezogen werden müssen. 